\title{
Model based automated diagnosis of bearing knock faults in internal combustion engines
}

\author{
Jian Chen ${ }^{1}$, Robert Bond Randall ${ }^{1}$ \\ School of Mechanical and Manufacturing Engineering, University of New South Wales, Syd- \\ ney 2052 Australia
}

\begin{abstract}
Intelligent diagnosis of bearing knock faults in Internal Combustion Engines (IC engines) was studied in this paper. Because of previous successful application of Artificial Neural Networks (ANNs) to the condition monitoring of rotating machinery, an ANN based automated diagnosis system was proposed for the diagnosis of big-end bearing knock faults in IC engines. It consists of three separate ANNs: a fault detection network, a fault localization network, and a fault severity identification network. In order to solve the problem that ANNs need a lot of data for training, a simulation model was built to simulate various degrees of bearing knock faults. The impact forces of the bearing with different clearance were simulated first, and then the accelerations at the measurement point on the engine block were calculated. A series of experiments were also carried out, and the results were used to evaluate and update the simulation model. It was also found that the squared envelope signals, rather the raw acceleration signals, have more useful diagnostic information. The extracted/selected amplitude features were used for fault detection and severity identification, and the extracted/selected phase features were used for fault localization. It is worth pointing out that because a saturating linear function was selected as the transfer function of the ANN for the fault severity identification stage, the networks can linearly classify the fault levels and the output agrees better with the real situation. All the networks were trained using simulated data and tested using experimental data. The final results have verified that the system could efficiently diagnose bearing knock faults, especially the accurate identification of the fault levels.
\end{abstract}

Keywords: Intelligent diagnosis, Bearing knock fault, Internal Combustion Engines, Artificial Neural Networks, simulation

\section{Introduction}

Big end bearing knock resulting from oversize clearance, due to excessive wear, is a common mechanical fault in Internal Combustion (IC) engines, but up to now, very limited work has been done on vibration based diagnosis of big end bearing knock faults. Additionally, when the vibration based diagnostic approach is used in real industrial situations, it normally requires an expert to interpret the analysis results from measured vibration signals. An Artificial Neural Network (ANN) based system

adfa, p. 1, 2011.

(C) Springer-Verlag Berlin Heidelberg 2011 
should be a potential solution to the problem of automated diagnostics. A critical issue with ANN applications is the network training, and it is neither feasible nor economical to be able to generate a sufficient number of different actual faults in seeded tests, to obtain sufficient experimental data for the network training. Therefore, modelling and simulation of different big end bearing knock faults is a viable way to generate a large amount of data for the network training.

Many studies have been devoted to the dynamic response of journal bearings with non-negligible clearance in the slider-crank mechanism. The majority have been focussed on the impact force of the small end bearing with clearance. In Schwab's thesis [1], considerable detail of the analytical modelling of joint clearances in mechanical systems with both dry contact and lubrication effects was discussed. Flores et al. [2-4] made many studies of the dynamic behaviours of lubricated revolute joints of the slider-crank mechanism. The effects of different eccentricity were also analysed. Based on the hydrodynamic bearing model with reciprocating motion developed by Bannwart et al. [5], Daniel and Cavalca [6] also studied the dynamic behaviour of the piston pin and the lubrication in the connecting rod-slider joint. The basic idea of their work is to use a computed numerical technique to iteratively solve the Lagrange equations and the lubrication equations. Even though all of these works were focussed on the small end bearing, a similar modelling principle can also be used for the study of the vibration characteristics of a big end bearing with clearance.

In this paper, a three-stage ANN based automated system is proposed for the diagnosis of big end bearing knock faults, including a fault detection stage, a fault localization stage and a severity identification stage. Simulation models were built to simulate various bearing knock faults in a Toyota engine, so as to provide sufficient inputs for the training of the ANN system. This simulation model for the impact force consists of two systems: a kinematic/kinetic system and a hydrodynamic system. The two subsystems are coupled by the lubrication oil force. After the impact forces with different clearance were simulated, the accelerations of the measurement point on the engine block surface were calculated. Meanwhile, a series of experiments with/without bearing knock faults were carried out to update and evaluate the simulation model. The networks were trained solely using simulated data and tested using real test data.

\section{Simulation of bearing knock fault}

\subsection{Mechanical Principle}

When an oversized clearance is introduced into the big end bearing, the Piston-con rod-crank system can be divided into two subsystems, one is the piston and connecting rod subsystem and other is the crank subsystem. The kinematic equations of the two subsystems can be built separately. The length of connecting rod is $b$. The length from the centre of the small end to the centre of gravity (CG) of the connecting rod is $b_{1}$ and the length from the centre of big end to the CG of the connecting rod is $b_{2}$. The radius of the crank is $R$. The coordinate of the crank journal centre is $\left(y_{c}, z_{c}\right)$ and the coordinate of the bearing centre is $\left(y_{b}, z_{b}\right)$. All the notations with subscript $c$ in the 
sections refer to the parameters of the crank journal and subscript $b$ to the parameters of the big end bearing. $F_{g}$ is the combustion force acting on the top of the piston. The lubricant force in the bearing can be represented by two components: horizontal $F_{y}$ and vertical $F_{z}$. The forces on the bearing and the journal have the same values but in opposite direction. $\theta$ is the crank angle and $\phi$ is the connecting rod angle. The small end bearing connecting the piston and connecting rod was assumed to be a perfect joint. For the whole system, there are three degrees of freedom: crank angular motion, connecting rod angular motion and the translational motion of the piston in the $Z$ direction.

The motion of the crank journal can be represented by the crank angular motion, and the moment balance equation on the journal is:

$$
I_{c} \ddot{\theta}=F_{y c} R \cos \theta+F_{z c} R \sin \theta-T_{\text {fric }}-T_{\text {load }}
$$

where $I_{c}$ is the moment of inertia of the crankshaft (plus flywheel), $T_{\text {fric }}$ is the friction and pumping torque of the engine, $T_{\text {load }}$ is the external load imposed on the engine.

The kinematic equation of the piston-connecting rod subsystem is more complicated and can be represented by the connecting rod angular motion and the translational motion of the piston in the $\mathrm{Z}$ direction. Similar to the work of Daniel et al. [6], the kinematic equations can be solved by Lagrangian mechanics.

$$
\left[\begin{array}{cc}
m_{p}+m_{b} & -b_{1} m_{b} \sin \phi \\
-b_{1} m_{b} \sin \phi & I_{b}+b_{1}^{2} m_{b}
\end{array}\right]\left[\begin{array}{l}
\ddot{z}_{p} \\
\ddot{\phi}
\end{array}\right]+\left[\begin{array}{cc}
0 & -\dot{\phi} b_{1} m_{b} \cos \phi \\
0 & 0
\end{array}\right]\left[\begin{array}{l}
\dot{z}_{p} \\
\dot{\phi}
\end{array}\right]=\left[\begin{array}{c}
F_{z b}-F_{g} \\
-F_{y b} b \cos \phi-F_{z b} b \sin \phi
\end{array}\right]
$$

where $m_{p}$ is the mass of the piston and $m_{b}$ is the mass of the connecting rod and $I_{b}$ is the moment of inertia of the connecting rod about its CG. $\dot{Z}_{p}$ and $\ddot{Z}_{p}$ are the velocity and acceleration of the piston in the $\mathrm{Z}$ direction.

\subsection{Hydrodynamic Principles}

Reynolds equation is a classic equation to solve for the hydrodynamic forces in the lubrication oil. Pinkus [7] has made a very detailed analysis of the hydrodynamic forces of different journal bearings.

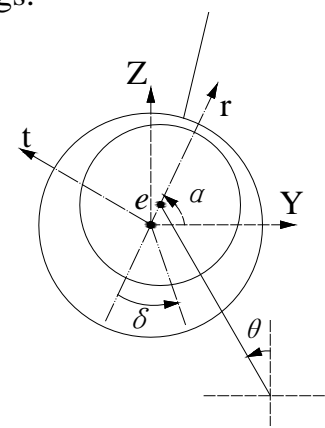

Fig. 1. The geometry of the big end bearing 
There are two coordinate systems, as shown in Figure 1. One is the global YZ system and the other is related to the eccentricity line $r$ system, based on the diameter through the point of minimum oil thickness. The $X$ direction is in line with the length of the bearing. $\alpha$ is the coordinate angle between the positive $r$ direction and the positive $Y$ direction. Because the load on the big end journal bearing is a variable rotating load, $\alpha$ should be a combination of attitude angle and rotation angle of the load. $e$ is the eccentricity. If $c$ is used to represent the radial clearance, the eccentricity ratio $\varepsilon$ can be defined as $e / c$. $\delta$ is the angle in the circumferential direction starting from the negative axis of $r$. The big end journal bearing is a typical dynamically loaded journal bearing, so the full Reynolds equation should be applied. It is very difficult to get the exact solution for Reynolds equation. However, Dubois \& Ocvirk [8] derived the solution for the infinitely short journal bearing in their work. The short bearing solution is reasonably valid for bearings with length to diameter ratio $\left(L_{b} / D_{b}\right)$ up to 0.5 . The length to diameter ratio of the big end bearings in the Toyota engine is less than 0.5 , so the oil film pressure distribution in the big end bearing can be solved as:

$$
\left.p=\frac{6 \mu c}{h^{3}}[\dot{\varepsilon} \cos \delta+\varepsilon(\dot{\alpha}-\bar{\omega}) \sin \delta)\right]\left(z^{2}-\frac{1}{4} L_{b}^{2}\right)
$$

Then the lubrication force can be integrated about the angle $\delta$. The normal force $F_{r}$ and tangential $F_{t}$ can be written as:

$$
\begin{aligned}
& F_{r}=-\int_{-L_{b} / 2}^{L_{b} / 2} \int_{\delta_{i}}^{\delta_{0}} p R_{b} \cos \delta d \delta d z \\
& F_{t}=-\int_{-L_{b} / 2}^{L_{b} / 2} \int_{\delta_{i}}^{\delta_{o}} p R_{b} \sin \delta d \delta d z
\end{aligned}
$$

where $\delta_{i}$ is the start angle and $\delta_{o}$ is the end angle of the integration. Gümbel [9] considered only the positive pressure section and assumed there is a $\pi$ oil film in the bearing. So the Gümbel boundary condition is more realistic for the force integration of short journal bearings. Frêne [10] has derived the analytical solution for the lubrication forces with Gümbel boundary condition for a short journal bearing and this can be used for the big end bearing. Alternatively, the forces can be solved by a numerical approach in the computer simulation software, just by integrating the positive pressure section about $\delta$.

Finally the force components in the global $Y Z$ coordinates can be derived by the transformation matrix about the angle coordinate $\alpha$ [7]:

$$
\left[\begin{array}{l}
F_{y} \\
F_{z}
\end{array}\right]=\left[\begin{array}{cc}
\cos \alpha & -\sin \alpha \\
\sin \alpha & \cos \alpha
\end{array}\right]\left[\begin{array}{l}
F_{r} \\
F_{t}
\end{array}\right]
$$

\subsection{The interaction between the kinematic/kinetic model and the lubrication model}

The interaction between the kinematic/kinetic model and the lubrication model is a closed loop (as shown in Figure 2). The whole simulation model was solved numerically in a step-by-step way. The inputs to the kinematic/kinetic system are the two lubrication force components: $F_{y}$ and $F_{z}$ and the outputs are the displacement parame- 
ters $z_{p}, \theta, \phi$ and the corresponding velocities $\dot{Z}_{p}, \dot{\theta}, \dot{\phi}$. Since the inputs to the lubrication system are the eccentricity ratio $\varepsilon$, the coordinate angle $\alpha$ and their derivatives. The outputs from the kinematic/kinetic system should be transformed by interaction equations before inputting into the lubrication system.

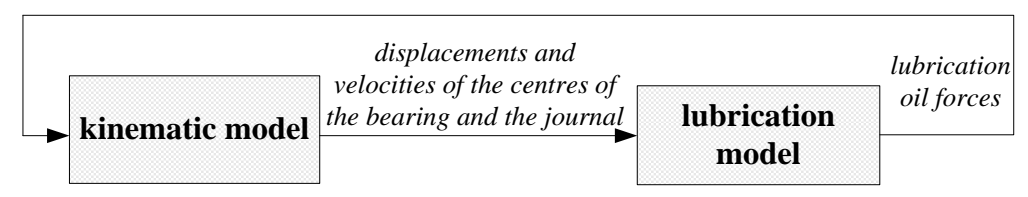

Fig. 2. Interaction between kinematic/kinetic system and lubrication system

\subsection{Simulation model}

All the models were built in Simulink. In the kinematic/kinetic system, the integration was solved by the ode45 method (Dortmund-prince). The solver used for the simulation is in variable time step mode, with minimal time step 1e-10 seconds and maximal time step 2e-7 seconds. All the output signals were sampled at a fixed rate $(200 \mathrm{kHz})$.

In order to compare the simulated results with experimental results, the physical parameters were from the tested Toyota 3SFE engine. The two stages of oversize clearance were selected as 0.2 and $0.4 \mathrm{~mm}$ for two-times normal clearance and fourtimes normal clearance, respectively. The engine speed was set at $3000 \mathrm{rpm}$ (315 $\mathrm{rad} / \mathrm{s}$ ). The combustion chamber pressure curves, corresponding to output loads of the engine at $50 \mathrm{Nm}, 80 \mathrm{Nm}$ and $110 \mathrm{Nm}$, were saved as look-up tables in the model. The chamber pressures were calculated using Wiebe's functions, for which the details can be obtained from the literature Refs. [11, 12].

The simulation model started at zero crank angle relative to TDC in the firing stroke. Based on the initial conditions, the displacements of the bearing centre and the journal centre were calculated first. Next the lubrication forces were calculated, and then the simulation loop continued. Even though the displacement of the piston in $Z$ direction was the same in the initial condition table for different clearances, it was found that the simulation reached a stable condition very quickly and the final results were independent of the initial displacement of the piston in the $Z$ direction.

\section{Experiments and signal processing}

The vibration tests on a Toyota 3SFE four-cylinder gasoline engine were carried out on an engine test rig. The firing sequence of the engine is 1-3-4-2. A proximity transducer was used to pick up the once-per-rev tacho signal, which corresponds to the Top Dead Centre (TDC) of cylinder 1 . The cylinder pressure was measured by a spark plug with integrated cylinder pressure sensor, and this could be used to update the cylinder pressure simulation model and select the tacho pulse corresponding to firing stroke. By controlling the dynamometer, the engine was controlled to operate at $3000 \mathrm{rpm}$ and three different load conditions: 50Nm, $80 \mathrm{Nm}, 110 \mathrm{Nm}$. Before all ma- 
chining works, the engine was dismantled and the clearances of the big end bearing were measured in an engine reconditioning workshop. The clearance was considered to be normal clearance. The journal of the crankshaft of the cylinder 2 was ground to increase the bearing clearance to two-time normal clearance (1st stage bearing knock fault) and four-time normal clearance (2nd stage bearing knock fault). An accelerometer was attached to the main bearing adjacent to the oversized big end bearing in the cylinder 2 and its measured vibration signal was used for the diagnosis of bearing knock faults.

The former work [13] has found that the vibration signals from bearing knock faults are typical second order cyclostationary signals and it was demonstrated that an appropriate signal processing approach for their diagnosis was squared envelope analysis of the vibration signals. The "fast kurtogram" developed by Antoni [14] was used to guide the selection of the optimum demodulation band $(400-4400 \mathrm{~Hz})$ before performing envelope analysis.

The comparison of the squared envelopes for the same speed/load but different clearance stages showed that the peak value has a substantial increase (about 10 times). The large increase also implies the possibility for the severity identification of bearing knock faults from envelope signals. The analysis of the squared envelope signals with bearing knock faults at different loads showed that the peak values of the squared envelope have no direct relationship with the increase of load. That is because the bearing knock impact force is mainly attributed to the inertia force of the piston and connecting rod components, not the combustion force. Note that near TDC these forces act in opposite directions.

\section{Comparison of experiment and simulation}

By adjusting the clearance, the impact forces with different degrees of bearing knock can be simulated. After the impact forces in the vertical and horizontal directions were simulated, their spectra were respectively multiplied by the corresponding measured Frequency Response Functions (FRFs) to get the impulse responses in the frequency domain. Afterwards, the accelerations in the time domain were obtained using the inverse Fourier transform. During the FRF measurement, according to the Maxwell's theory of reciprocity, an excitation force was applied to the surface (measurement) point of the engine block and the acceleration was picked up on the crank journal. As mentioned in previous section, the measured vibration signal is sampled at $25600 \mathrm{~Hz}$, but the measured FRFs cut off at $6400 \mathrm{~Hz}$. Therefore, compared to the experimental vibration signal, less high frequency components can be found in the simulated vibration signals and the amplitude of the simulated vibration is smaller than the experiments. However, the diagnostic information for the mechanical faults lies in the envelope of the acceleration signal. Before taking the envelope, the experimental acceleration signals were filtered by a band pass filter $(400-4400 \mathrm{~Hz})$, so most of the high frequency content was removed in the signal processing.

As mentioned before, the target of the simulations here is to produce the envelope signals as accurately as possible rather than the raw acceleration signals, so the enve- 
lopes of the simulated vibration signals were calculated. The simulated squared envelopes with $2^{\text {nd }}$ stages of bearing knock faults are shown in Figure 3, and the zero degree point in the crank angle corresponds to the TDC of cylinder 1, firing stroke. It was found that the peak value of the simulated amplitude with the $2^{\text {nd }}$ stage bearing knock fault is smaller than for the experiment, the ratios of change (about 10 times) of the peak values from the normal condition to the $1^{\text {st }}$ stage and to the $2^{\text {nd }}$ stage are adequate for the fault detection and the identification of the fault severity.
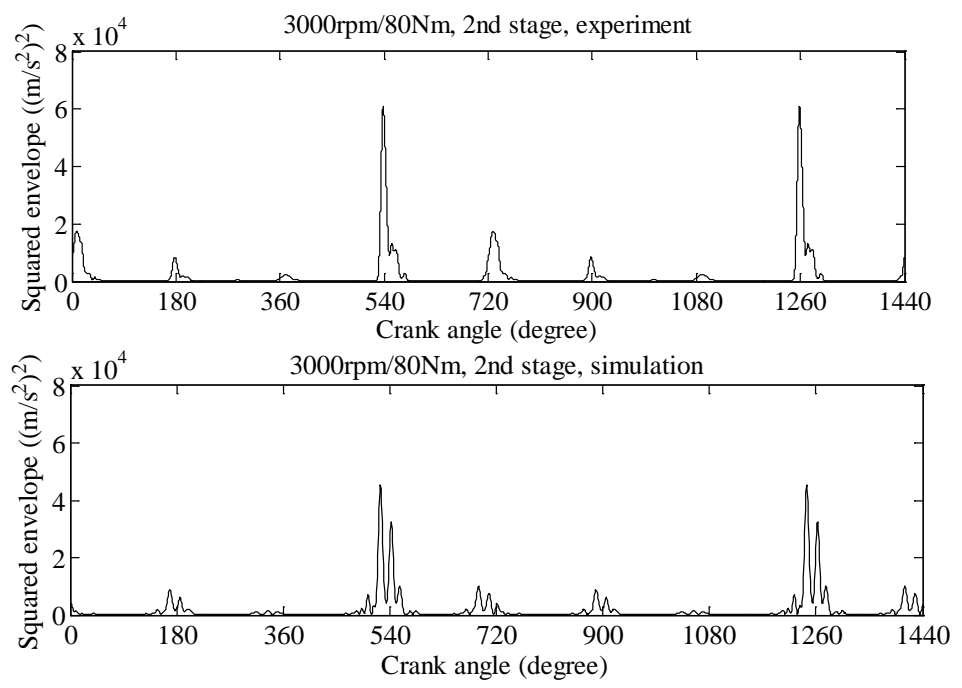

Fig. 3. Envelope signals with the $2^{\text {nd }}$ stage bearing knock fault at 3000rpm/80Nm

\section{Automated diagnosis of bearing knock faults}

As the former work demonstrated [13,15-16], the amplitudes of the Fourier coefficients of the squared envelope signal were potential features to detect the bearing knock fault and identify the severity, and the phase provided potential features to classify which cylinder had the fault. Due to the transient characteristics, the amplitude and phase of the first 40 harmonics of the envelope signal were considered. But not all the candidate features are useful for the pattern recognition of mechanical faults. A GA-based advanced feature selection method was applied to select the best amplitude features for the diagnosis of the mechanical faults and the detail can be obtained in reference [16]. As analysed in reference [16], when the faults occur in a certain cylinder for different speed/load conditions, if the phases of the nth harmonic of all cases are grouped or near fixed, it implies that this phase is a potential feature for identifying the localization of faults. So the phase feature selection is based on a principle of minimal scattering ratio. The bearing knock faults in different cylinders were also simulated. 
The simulated signal with bearing knock faults is deterministic, but in reality the measured signals have deviations. Based on the range of variations in the measurements, variations were instituted in the simulated signals to create representative cases for the ANN training. Separate networks were developed for the three stages of the diagnosis problem. The first stage is the fault detection stage where the inputs to the networks are the selected amplitude features. In the second stage, the neural network localizes which cylinder has faults and the inputs to the network are the selected phase features. In the third stage, based on the detection and location results, using selected amplitude features, the severity of the faults is predicted. MLP (Multi Layer Perceptron) networks were used in the detection stage (MLP1) and severity/prognosis stage (MLP2). The log sigmoid function was used as the transfer function of MLP1, this is because it can efficiently classify all cases into two groups (converging to two boundary conditions); 0 for normal condition and 1 for bearing knock fault. Different from our former work [17], the saturating linear function, rather than nonlinear log sigmoid function, was introduced into MLP2 as its transfer function. This saturating linear function is linear in the interval $(0,+1)$ and saturates outside this interval to 0 or +1 . It returns the value of $x$ truncated into the range 0 to 1 . So the output of MLP2 can be any value linearly distributed from 0 to 1 , for instance, 0.5 represents two-times normal clearance, with 1 for four-times normal clearance and 0.75 for three-times normal clearance. Therefore, the output results of severity identification agree more logically with the real situation. The upper limit of 1 could be set to agree with the maximum viable value of clearance. PNNs (Probabilistic Neural Networks) were used to identify which cylinder has faults. The outputs of the PNN for the misfire diagnostics are the integer numbers $1,2,3$ and 4, which directly indicate the cylinder number.

MLP1was trained purely using the simulation data. There are 120 cases, including 72 cases with bearing knock faults and 48 cases without bearing knock faults. MLP1 was tested using 37 experimental cases; those from cases 1 to 10 are with bearing knock faults and those from cases 11 to 37 are with normal bearing clearance (but from 32 to 37 include piston slap faults). The final results of MLP1 are shown in Figure 4. It can be seen that the MLP1 100\% correctly detected the bearing knock faults (using 0.5 as a threshold), though one piston slap case approached $60 \%$ of the threshold.

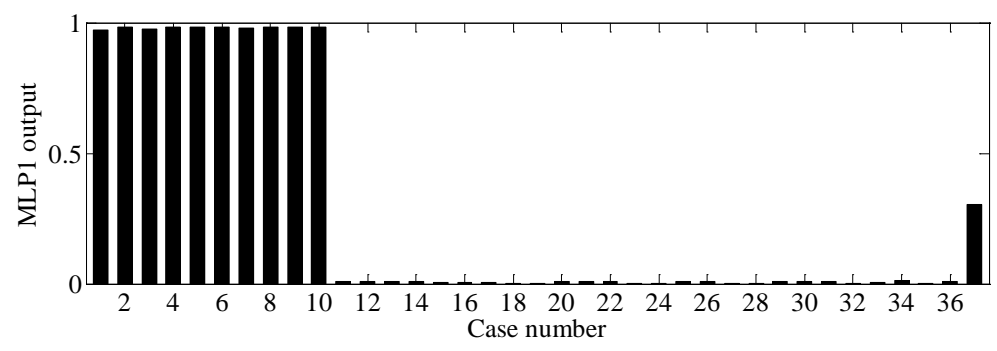

Fig. 4. Output of MLP1 for the bearing knock faults

After the detection stage, the number of the training cases for the PNN reduced to 72 and the number of the test cases reduced to 10 . The final results show that all output numbers from the PNN are 2, so the PNN 100\% correctly identified which 
cylinder had the bearing knock faults. Meanwhile, the severity identification became more specific and the number of the training cases for MLP2 also reduced. The output of MLP2 is shown in Figure 5. It can be seen that the MLP2 also got $100 \%$ correct results for the severity identification, and especially for two-times normal clearance (output 0.5 ), the errors are very small.

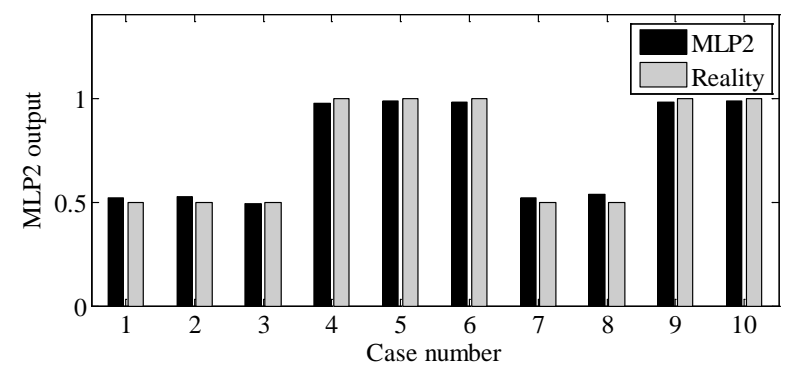

Fig. 5. Output of MLP2 for the bearing knock faults

\section{Conclusion}

A new simulation model, which can simulate various degrees of bearing knock faults in engines, was developed to create sufficient data for the training of the ANNs. In order to update and evaluate the simulation model, a series of experiments were carried out on an engine test rig, including normal condition and with two stages of bearing knock faults. The amplitude and phase features were extracted and selected from the processed squared envelope signals. The amplitude features were used for fault detection and fault severity identification; the phase features were used to classify which cylinder had faults. The three stage ANN system was trained purely using the simulation data and tested using the experimental data. The results have demonstrated that the developed system could efficiently diagnose different bearing knock faults, and in particular could accurately identify the severity levels of the faults. It is worth pointing out that because the simulation models are based on the thermodynamic and mechanical principles of IC engines, the proposed modelling method and diagnostic system can be adapted for any engine.

\section{Acknowledgements}

The authors would like to convey special gratitude to the Australian Research Council and LMS International for sponsoring this research under Linkage Project LP0883486. 


\section{References}

1. Schwab A.L.: Dynamics of flexible multibody systems, small vibrations superimposed on a general rigid body motion. PhD Thesis, Delft University of Technology, Netherlands (2002)

2. Flores P., Ambrósio J., Claro J. C. P., Lankarani H. M. and Koshy C. S.: Lubricated revolute joints in rigid multibody systems. Nonlinear Dynamics, vol.56, no.3, 277-295 (2009)

3. Flores P., Ambrósio J., Claro J. C. P., Lankarani H. M. and Koshy C. S.: A study on dynamics of mechanical systems including joints with clearance and lubrication. Mechanism and Machine Theory, vol. 41, no. 3, 247-261 (2006)

4. Flores P., Lankarani H. M., Ambrósio J. and Claro J. C. P.: Modeling lubricated revolute joints in multibody mechanical systems. Proceedings of the Institution of Mechanical Engineers, Part K: Journal of Multi-body Dynamics, vol.218, 183-190 (2004)

5. Bannwarta A. C., Cavalca K.L. and Daniel G. B.: Hydrodynamic bearings modeling with alternate motion. Mechanics Research Communications, vol.37, no.6, 590-597 (2010)

6. Daniel G. B., Cavalca K. L.: Analysis of the dynamics of a slider-crank mechanism with hydrodynamic lubrication in the connecting rod-slider joint clearance. Mechanism and Machine Theory, vol.46, no.10, 1434-1452 (2011)

7. Pinkus, O. and Sternlicht, S. A., "Theory of Hydrodynamic Lubrication”, McGraw-Hill Press, New York, USA (1961)

8. Dubois G. B. and Ocvirk F. W., "Analytical derivation and experimental evaluation of short bearing approximation for full journal bearings", NACA report 1157, Washington, USA (1953)

9. Gümbel L. K. R. "Vergleich der ergebnisse der rectinerischen Behandling des Lagerschmierungsproblem mit neueren Versuchsergebnissen”, Monatsbl. Berliner Bez. Ver. Dtsch. Ing., 125-128 (1921)

10. Frêne, J., Nicolas, D., Degneurce, B., Berthe, D. and Godet, M., "Hydrodynamic Lubrication - Bearings and Thrust Bearings”, Elsevier, Amsterdam, Netherlands (1997)

11. Chen J., Randall R. B., Peeters B., Van der Auweraer H. and Desmet W.: Automated misfire diagnosis in engines using torsional vibration and block rotation. COMADEM2012, Huddersfield, UK, 18-20th June (2012)

12. Chen J. and Randall R. B.: Simulating faults in IC engines. the 19th International Congress on Sound and Vibration, Vilnius, Lithuania, 8-12th July (2012)

13. Chen J., Randall R. B., Peeters B., Desmet W., Van der Auweraer H.: Artificial neural network based fault diagnosis of IC engines. Key Engineering Materials, vol. 518, 47-56 (2012)

14. Antoni, J.: Fast computation of the kurtogram for the detection of transient faults. Mechanical Systems and Signal Processing, vol.21, no.1, 108-124 (2007)

15. Chen J., Randall R. B.: Simulating bearing knock in an IC engine. the 20th International Congress on Sound and Vibration, 7-11th July 2013, Bangkok, Thailand

16. Chen J., Randall R. B., Peeters B., Desmet W. and Van der Auweraer H.: Neural network based diagnosis of mechanical faults in IC engines. IMechE Tenth International Conference on Vibrations in Rotating Machinery, 11-13rd September 2012 London, UK

17. Chen J., Randall R. B.: Automated diagnosis system for mechanical faults in IC engines. CM/MFPT 2013, 17-20th June 2013, Kraków, Poland 